\title{
Yes-associated protein reacts differently in vascular smooth muscle cells under different intensities of mechanical stretch
}

\author{
Xinhao Wang ${ }^{1}$, Xiangdang Liang ${ }^{2}$, Lujun $\mathrm{Zhou}^{3}$, Shen $\mathrm{Liu}^{2}$, Zhuoqun Fang ${ }^{2}$, Chuanzhong $\mathrm{Hu}^{2}$, \\ Yigong $\mathrm{Hou}^{2}$, Zhanshe Guo ${ }^{4}$ \\ ${ }^{1}$ Department of Vascular and Endovascular Surgery, Chinese PLA General Hospital, Beijing, China \\ ${ }^{2}$ Department of Orthopedics, Chinese PLA General Hospital, Beijing, China \\ ${ }^{3}$ State Key Laboratory of Brain and Cognitive Sciences, Institute of Biophysics, Chinese Academy of Sciences, \\ Beijing, China \\ ${ }^{4}$ School of Instrument Science and Optoelectronic Engineering, Beihang University, Beijing, China
}

Correspondence to: Xiangdang Liang; email: Ixd301@263.net, https://orcid.org/0000-0002-1318-9370

Keywords: Hippo pathway, YAP, mechanical stretch, proliferation, apoptosis

Received: December 18, 2020 Accepted: October 18, 2021

Published: January 4, 2022

Copyright: (C) 2021 Wang et al. This is an open access article distributed under the terms of the Creative Commons Attribution License (CC BY 3.0), which permits unrestricted use, distribution, and reproduction in any medium, provided the original author and source are credited.

\section{ABSTRACT}

Vascular smooth muscle cells (VSMCs) are stromal cells of the vascular wall and are continually exposed to mechanical signals. The loss of VSMCs is closely related to the occurrence of many vascular diseases, such as aortic aneurysms and aortic dissection. The proliferation and apoptosis of VSMCs are mechanically stimulated. Yesassociated protein (YAP), one of the core components of the Hippo pathway, plays a key role in the response of VSMCs to mechanical signals. In this study, we tested the impact of different intensities of mechanical stretch on the proliferation and apoptosis of VSMCs, as well as YAP. We tested VSMCs' proliferation and apoptosis and YAP reaction via immunocytochemistry, western blotting, CCK-8 and flow cytometric analysis. We found that $10 \%$ elongation could increase the phosphorylation of YAP and prevent it from entering the nucleus, as well as inhibit cell proliferation and promote apoptosis. However, 15\% elongation reduced YAP phosphorylation and promoted its nuclear entry, thereby promoting cell proliferation and inhibiting apoptosis. Accordingly, YAP knockdown suppressed the phenotype of VMSCs induced by $15 \%$ elongation. Taken together, YAP regulates proliferation and apoptosis of VSMCs differently under different intensity of mechanical stretch. Mechanical stretch with appropriate intensity can promote the proliferation and inhibit apoptosis of VSMCs by activating YAP.

\section{INTRODUCTION}

Aortic aneurysms are associated with aortic dissection and rupture. There is currently no effective treatment to prevent or cure aortic aneurysms. At present, both the pathogenesis and pathophysiology of ascending aortic aneurysms are not entirely clear. Vascular smooth muscle cells (VSMCs) have been recognized as the most important factor in the development of ascending aortic aneurysms. Aortic aneurysms can occur due to loss of VSMCs in the media layer of the aortic wall, leading to progressive aortic dilation [1-3]. We observed an interesting phenomenon in clinical work where the aneurysm or dissection remodeling varies from site to site, which may be due to differences in the mechanical stimuli to which different sites are exposed.

Aortic walls are subjected to various mechanical stimuli from the bloodstream, such as shear and mechanical stretch. Stretch sensing is generally known as an integrin-mediated pathway, which is coupled to cell contractile activity, and thus shares many mechanotransduction pathways with the rigidity sensing process in translating mechanical stimuli into intracellular biochemical signals [4-6]. The relationship between mechanical stretch and cell proliferation/apoptosis has 
been extensively studied [7-11]. VMSCs experience mechanical stimuli during growth and differentiation and transduce these stimuli into biochemical signals that in turn regulate cell responses to the imposed forces. The effect of cyclic stretch is recognized as an important regulator of the development and pathological abnormalities of aortic walls. Under physiological conditions, the aorta undergoes approximately $10 \%$ circumferential stretch during systole. This number increases to approximately $20 \%$ under conditions of hypertension. The rate of apoptosis of VSMCs under $20 \%$ circumferential stretch is higher than that under $10 \%$ stimulation; how VSMCs change under 10-20\% stretch remains uncertain [12].

The Hippo pathway plays an important role in the cell's reaction to mechanical stretch [13-17]. It was first defined in Drosophila by genetic mosaic screening following identification of a loss-of-function mutation of Hippo that led to a strong overgrowth phenotype [18]. As the major downstream effector of the Hippo pathway, YAP/TAZ mediates major physiological functions therein. MST1/2, Sav1, LATS1/2, and Mob1 constitute a kinase cascade that eventually phosphorylates YAP/TAZ and promotes its binding with 14-3-3 and cytoplasmic retention $[19,20]$. YAP/TAZ has been identified as the sensor and mediator of mechanical cues arising according to the rigidity of the extracellular matrix, cell geometry, cell density, and the status of the actin cytoskeleton $[14,15$, 21, 22]. Among them, YAP, as an important factor in mechanical signal transduction, controls cell survival and proliferation by combining with DNA-binding transcription factors to induce gene expression (Hippo pathway in organ size control, tissue homeostasis, and cancer). YAP inhibits the expression of smooth muscle differentiation genes, and at the same time promotes smooth muscle proliferation and migration in vitro and in vivo, and plays a novel comprehensive role in smooth muscle phenotype regulation (The induction of yesassociated protein expression after arterial injury is crucial for smooth muscle phenotypic modulation and neointima formation). Therefore, these evidences indicate that YAP is a key molecule in the regulation of VSMC phenotype. Rho-ROCK is the signaling pathway upstream of Hippo. The Rho-ROCK signaling pathway inhibits Hippo pathway activity [23, 24]. Rho-ROCK is affected by mechanical stress and regulates the proliferation of VSMCs. However, the influence of different intensities of mechanical stretch on the Hippo pathway, and the role of Rho-ROCK in this mechanism, remain unclear.

In our study, we placed VSMCs under different intensities of mechanical stretch in vitro. We aim to find out that how the Hippo pathway and cell proliferation of
VSMCs changes under mechanical stretch from $0 \%$ to $15 \%$ elongation. Based on our study, we wish to achieve a better understanding of the relationship between different intensities of mechanical stretch and cell proliferation of VSMCs.

\section{MATERIALS AND METHODS}

\section{VSMCs isolation and culture}

Sprague-Dawley rats (male, 200-250 g) were purchased from the animal experimental center of Academy of Military Medical Sciences of PLA (Beijing, China) and housed under the specific pathogen-free (SPF) conditions (temperature, $23 \pm 2^{\circ} \mathrm{C}$; relative humidity, $65 \% \pm 5 \% ; 12 \mathrm{~h} / 12 \mathrm{~h}$ light / dark cycle, 07:00-19:00) with free access to food and water for 3 days. SD rats were anesthetized with isoflurane, and then VSMCs were isolated from thoracic aorta using the explanting technique [25]. and cultured in Dulbecco's modified Eagle's medium (DMEM; Gibco, Grand Island, NY, USA) containing $10 \%$ fetal bovine serum (FBS; Gibco, Grand Island, NY, USA), $100 \mathrm{U} / \mathrm{mL}$ penicillin, and 100 $\mu \mathrm{g} / \mathrm{mL}$ streptomycin at $37^{\circ} \mathrm{C}$ in a humidified atmosphere of $5 \% \mathrm{CO}_{2}$. The medium was changed every $2 \mathrm{~d}$, and cells were passaged by treatment with a $0.05 \%$ trypsin-EDTA solution. The cells were used between passages 3 to 8 . All animal experimental procedures were in accordance with the National Institutes of Health's Guide for the Care and Use of Laboratory Animals and were approved by the Animal Care and Use Committee of the Military Medical Sciences of PLA, as well as the Animal Laboratory Administration Center and Ethics Committee of the Military Medical Sciences of PLA.

\section{Cyclic stretch stress on VSMCs}

VSMCs were plated in 6-well plates (Flexcell International Corp., Hillsborough, NC, USA) coated by type I collagen (Solarbio, Beijing, China) at a concentration of $3 \times 10^{5}$ cells $/ \mathrm{mL}$. After $24 \mathrm{~h}$ attachment, the cells were synchronized by DMEM with $10 \%$ FBS for another $24 \mathrm{~h}$ and then applied to cyclic stretch produced by FX-5000T Tension System (Flexcell International Corp., Hillsborough, NC, USA) with 10,15 , and $20 \%$ elongation at a frequency of $1 \mathrm{~Hz}$ (60 cycles/min), and the duration of cyclic stretching forces for $24 \mathrm{~h}$.

\section{YAP SiRNA}

Experimental 1 consisted of the following groups: VSMCs, VSMCs+YAP siRNA NC (TTCTCCGAACG TGTCACGT), VSMCs+YAP siRNA 1 (ACAGCAGGA GTTATTTCGG), VSMCs+YAP siRNA 2 (GACCTCT 
TCTGGTCAGAGA), and VSMCs+YAP siRNA 3 (ATCACAATGATCAGACAAC). Cells were inoculated in 6-well plates at $2 \times 10^{6}$ cells/well and cultured overnight. Each well was diluted to $250 \mu \mathrm{L}$ of Opti-MEM I Reduced Serum Medium by adding 100 pmol of siRNA. Lipofectamine 2000 (5 $\mu \mathrm{L}$; Life Technologies, Carlsbad, CA, USA) was added to $250 \mu \mathrm{L}$ of Opti-MEM I Reduced Serum Medium and incubated for $5 \mathrm{~min}$. Then, the siRNA solution was added to the Lipofectamine 2000 solution and incubated for $20 \mathrm{~min}$. The culture medium in the cell culture plate was aspirated and $1.5 \mathrm{~mL}$ fresh medium was added. The siRNA solution was added to the samples and cultured in a $5 \% \mathrm{CO}_{2}$ incubator at $37^{\circ} \mathrm{C}$ for $48 \mathrm{~h}$. Western blotting was used to assess the results and VSMCs were reclassified according to the obtained results.

Experimental 2 consisted of the following groups: VSMCs control+0\% elongation, VSMCs YAP shRNA+0\% elongation, VSMCs control+10\% elongation, VSMCs YAP shRNA+10\% elongation, VSMCs control $+15 \%$ elongation, and VSMCs YAP shRNA $+15 \%$ elongation. shRNA using the best one of the previous experiment which is siYAP1. VSMCs were cultured as described above. Cells were infected with the viruses at a multiplicity of infection of 50, and control groups were transfected with lentiviruses containing control sequences.

\section{Inhibition of the Rho/ROCK pathway}

Experimental 3 consisted of the following groups: VSMCs $+0 \%$ elongation, VSMCs $+0 \%$ elongation+Y27632, VSMCs+10\% elongation, VSMCs $+10 \%$ elongation $+Y 27632, \quad$ VSMCs $+15 \%$ elongation, and VSMCs+15\% elongation+Y27632. VSMCs were cultured as described above. The stretching frequency was $1 \mathrm{~Hz}$ and the stretching times were set to 1 $\mathrm{h}$ and $6 \mathrm{~h}$. The final concentration of Y27632 $(3 \mu \mathrm{mol} / \mathrm{L}$; T1870; TargetMol, Boston, MA, USA) treatment was 50 $\mu \mathrm{M}(0.5 \%$ dimethyl sulfoxide concentration) .

\section{Flow cytometric analysis and CCK-8 assay}

VSMCs from each group were stained with annexin Vfluorescein isothiocyanate to determine the number of apoptotic cells. And another samples were fixed with $75 \%$ ethanol and treated with RNase to analyze the cell cycle. Then, cell nuclei were stained with propidium iodide (Molecular Probes, Eugene, OR, USA), and VSMCs were analyzed using a FACSCalibur flow cytometer and Cell Quest software (Becton Dickinson, Franklin Lakes, NJ, USA).

VSMCs were inoculated in 6-well culture plate. Cell counting kit-8 (CCK-8; DOJINDO, Kumamoto, Japan) solution was mixed with serum-free medium in a $1: 10$ ratio $(\mathrm{v} / \mathrm{v})$. The mixture was added at $100 \mu \mathrm{L}$ per well and incubated at $37^{\circ} \mathrm{C}$ and $5 \% \mathrm{CO}_{2}$ for $1 \mathrm{~h}$. Absorbance at $450 \mathrm{~nm}$ was measured using an enzyme marker.

\section{Immunocytochemistry analysis}

VSMCs from each group were fixed in $4 \%$ paraformaldehyde for $20 \mathrm{~min}$, and permeabilized with $0.2 \%$ Triton $\mathrm{X}-100$ for $10 \mathrm{~min}$ at room temperature. Each sample was dripped with 3\% BSA blocking solution and sealed at room temperature for $30 \mathrm{~min}$. Then, samples were incubated with primary antibody to YAP (bs-3605R; Bioss, Los Angeles, CA, USA) and fluorescent CY3 goat anti-rabbit IgG secondary antibody (rhodamine-labeled, BA1036; Boster Biological Technology, Pleasanton, CA, USA). The nuclei were counterstained with 4', 6-diamidino-2phenylindole (DAPI; 1:500). The samples were observed under the confocal laser scanning microscopy.

\section{Western blotting}

Treated VSMCs were harvested in lysis buffer using protease inhibitors, and total protein was extracted and quantified using a BCA protein concentration kit according to the manufacturer's instructions. Proteins were separated by sodium dodecyl sulfate polyacrylamide gel electrophoresis (SDS-PAGE) and transferred to polyvinylidene fluoride (PVDF) membranes. The membranes were blocked with $5 \%$ BSA in Tris-buffered saline with Tween-20 (TBST) for $2 \mathrm{~h}$ at room temperature, and incubated overnight at $4^{\circ} \mathrm{C}$ with primary antibody (anti-YAP [bs-3605R; Bioss], anti-p-YAP [ab76252; Abcam, Cambridge, UK], anti-cyclin D1 (ccnd1) [ab134175; Abcam], antiLats [ab243656; Abcam, Cambridge, UK], anti-p-Lats [bs-4082R; Bioss], anti-Rho [ab32046; Abcam, Cambridge, UK], anti-ROCK [ab45171; Abcam, Cambridge, UK], and anti- $\beta$-actin [bs-0061R; Bioss]). The membranes were incubated with IRDye $800 \mathrm{CW}$ goat anti-rabbit IgG $(\mathrm{H}+\mathrm{L})$ (926-32211; Licor, Lincoln, $\mathrm{NB}$, USA) at room temperature for $1 \mathrm{~h}$. Bands were detected using the chemiluminescent imaging system.

\section{Statistical analysis}

One-way analysis of variance was performed in SPSS software (ver. 19.0; SPSS Inc., Chicago, IL, USA) to evaluate group differences. Data are expressed as means \pm standard deviation, and $P$ values $<0.05$ were considered to indicate statistical significance.

\section{Availability of data and materials}

All data generated or analyzed during this study are included in this published article. 


\section{RESULTS}

\section{Mechanical stretch intensity influences the proliferation and apoptosis of VSMCs}

To determine the changes in proliferation and apoptosis of VSMCs under different intensities of mechanical stretch, we applied 0,10,15, and 20\% elongation forces to VSMCs (Figure 1). We used the CCK-8 assay to assess cell proliferation and flow cytometric analysis to detect apoptosis and analyze the cell cycle. The 10\% and $20 \%$ elongation forces increased the rate of apoptosis and downregulated VSMCs proliferation (Figure 1). Meanwhile, 15\% elongation produced the opposite results, i.e., decreased the rate of apoptosis and upregulated VSMCs proliferation (Figure 1). 10\% and $20 \%$ of the stretch-induced S phase cells reduce might inhibit cell proliferation, while $15 \%$ stress-induced S phase cells increase might promote cell proliferation (Figure 1).

\section{Effects of different intensities of mechanical stress on YAP}

To test whether YAP was involved in the effects of mechanical stress on VSMCs, we first performed immunofluorescence staining to determine the intracellular localization of YAP. YAP was both in the nucleus and the cytoplasm under 0\% elongation (Figure 2A-2C). YAP was primarily cytoplasmic under $10 \%$
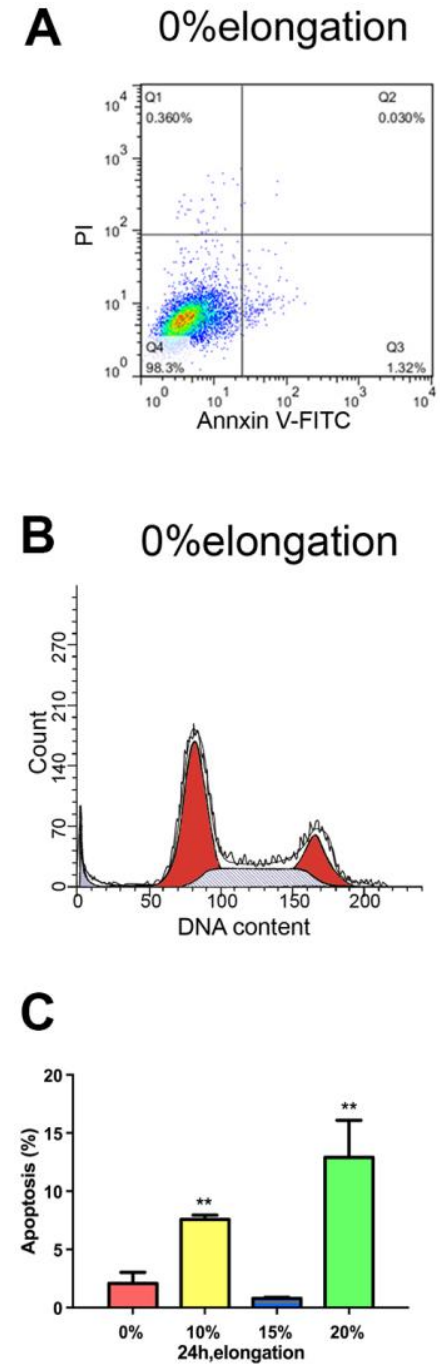

$10 \%$ elongation

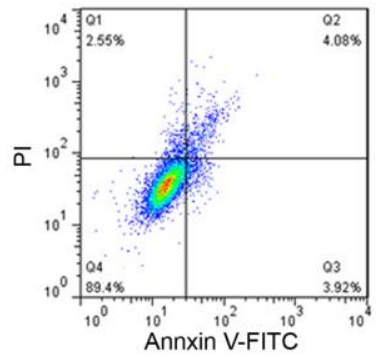

$10 \%$ elongation

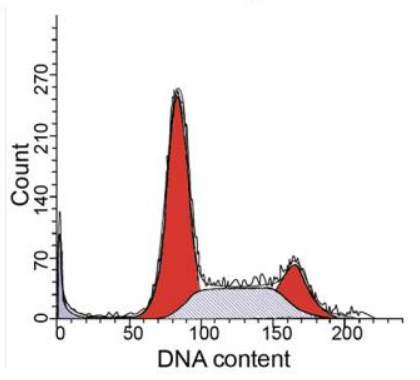

D

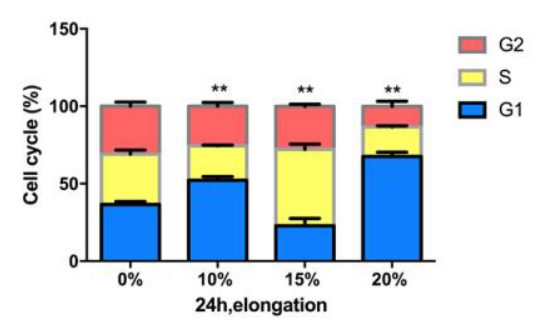

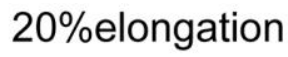
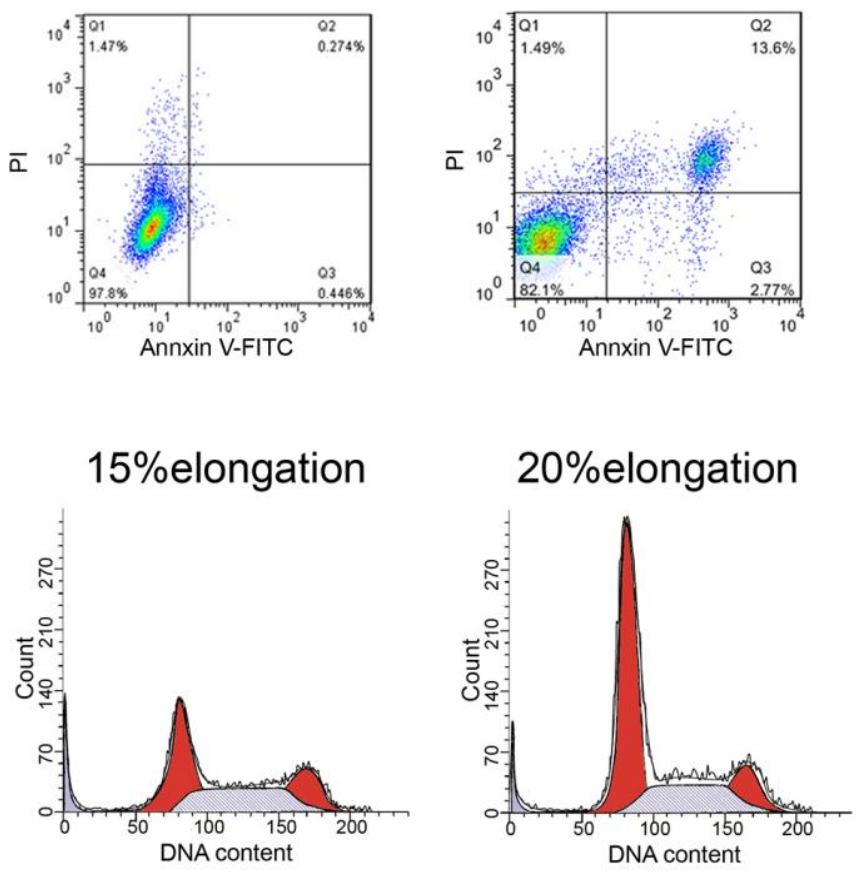

E

Figure 1. Mechanical stretch intensity influences the proliferation and apoptosis of VSMCs. (A) Apoptosis of VSMCs was detected by flow cytometry. (B) The cell cycle of VSMCs was detected by flow cytometry. (C) Quantitative analysis of apoptosis in VSMCs. (D) Quantitative analysis of the cell cycle in VSMCs. (E) The proliferation of VSMCs was detected by Cell Counting Kit 8 (CCK-8). Values are expressed as means \pm SD. ${ }^{*} P<0.05,{ }^{* *} P<0.01$, compared with $0 \%$ elongation group. 
elongation conditions, whereas YAP was primarily nuclear with $15 \%$ elongation (Figure 2A-2C). Intracellular localization of YAP was linked to YAP phosphorylation, so we determined the level of YAP phosphorylation in each group. YAP phosphorylation was upregulated under $10 \%$ elongation, while phosphorylation of YAP remained low under both $0 \%$ and $15 \%$ elongation and being the lowest under 15\% elongation (Figure 2D). Because nuclear localization of YAP was linked to the expression of ccnd1, we also assessed the protein levels of ccnd 1 in each group at the same time. The protein levels of ccnd1 varied according to the phosphorylation level of YAP. Compared with $0 \%$ elongation, expression of ccnd1 decreased with $10 \%$, and increased with $15 \%$ elongation

A

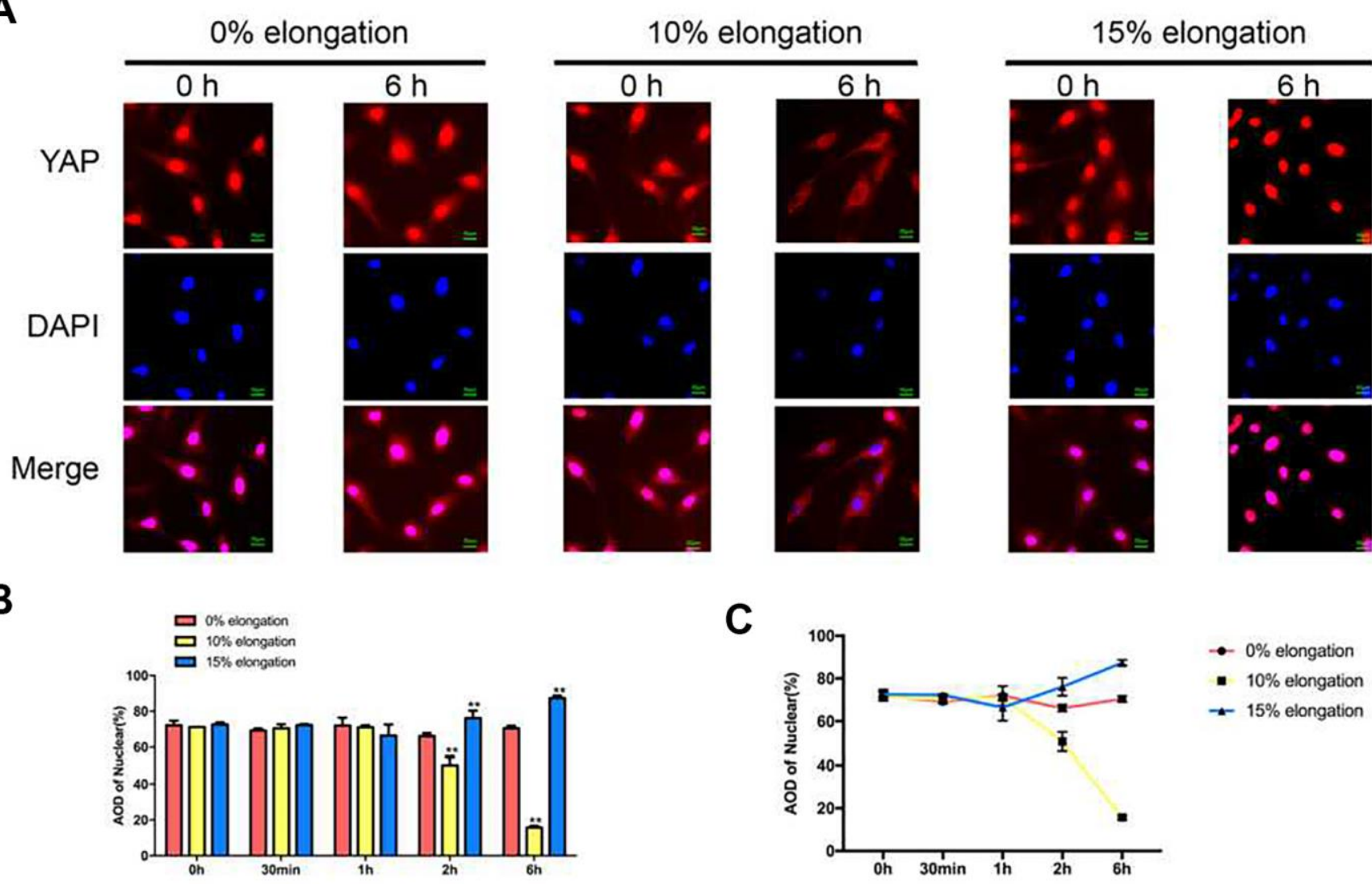

D

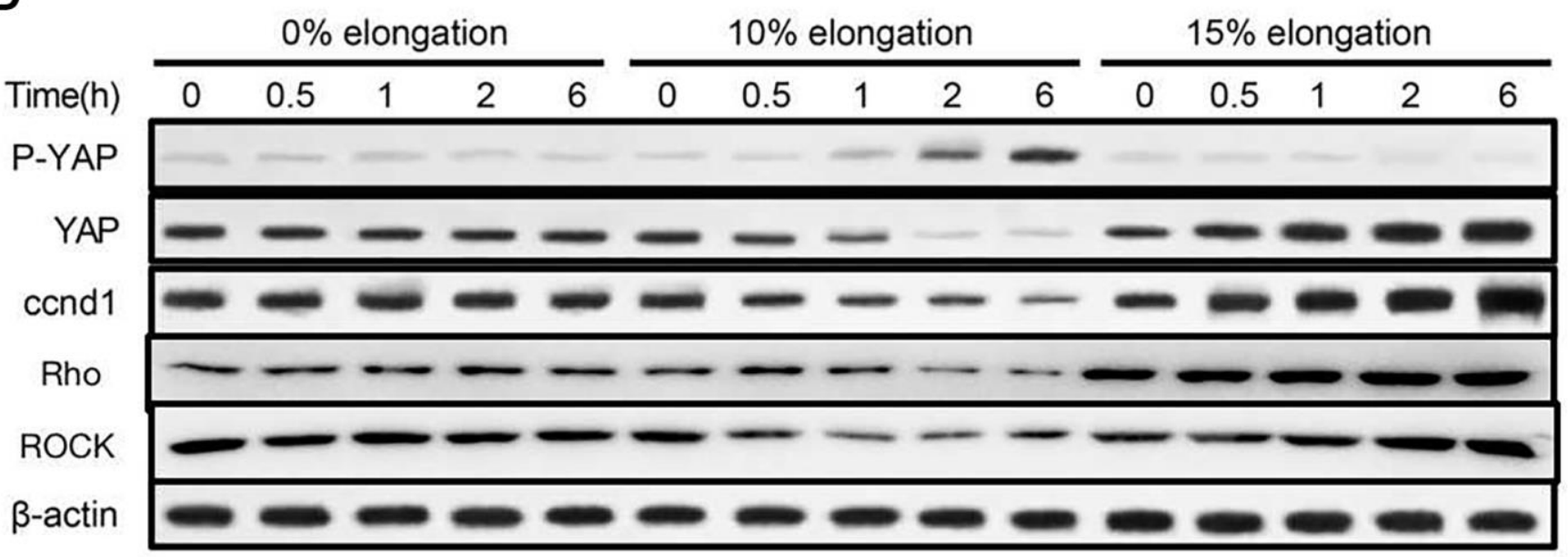

Figure 2. Effects of different intensities of mechanical stress on YAP. (A) The YAP localization of VSMCS was detected by immunocytochemistry. (B, C) Quantification of nuclear YAP amount in VSMCs. (D) The levels of phosphorylated YAP and cyclin D1 (ccnd1) in VSMCs were detected by western blotting. Values are expressed as means \pm SD. ${ }^{* *} P<0.01$, compared with $0 \%$ elongation group. 
(Figure 2D). Next, we tested the expression of key protein molecules in the Rho-ROCK signaling pathway. Similarly, compared with $0 \%$ elongation, expression of Rho and ROCK decreased with $10 \%$, and increased with $15 \%$ elongation (Figure 2D).

\section{Knockdown of YAP impairs proliferation and apoptosis of VSMCs under different intensities of mechanical stretch}

To determine the effects of the Hippo pathway in VSMCs, we knocked down YAP by siRNA. We tested the efficiency of different YAP-targeting siRNAs using western blotting to generate optimal knockdown of YAP. Our results showed that YAP siRNA1 (ACAGCAGGAGTTATTTCGG) resulted in the greatest knockdown of YAP (Figure 3A). To test the effects of YAP knockdown on VSMCs proliferation, apoptosis, and the cell cycle under different intensities of mechanical stimulation, we performed CCK-8 assays and flow cytometry. The CCK-8 assays indicated that cell proliferation was inhibited following YAP knockdown in each group (Figure 3F). Compared with $0 \%$ elongation, VSMCs proliferation was decreased in the absence of YAP with $10 \%$ elongation, but increased with 15\% elongation (Figure 3F). Following YAP knockdown, VSMCs showed a similar rate of proliferation under the different mechanical stimulations, but with smaller amplitudes (Figure 3F). Flow cytometry analysis demonstrated that apoptosis of VSMCs was increased following YAP knockdown under all stretch intensities (Figure 3B, 3C). The rate of apoptosis of VSMCs was significantly increased following YAP knockdown under $10 \%$ elongation (Figure 3B, 3C). Moreover, flow cytometry revealed that YAP knockdown kept more VSMCs in the G0/G1 phase under the same intensity of
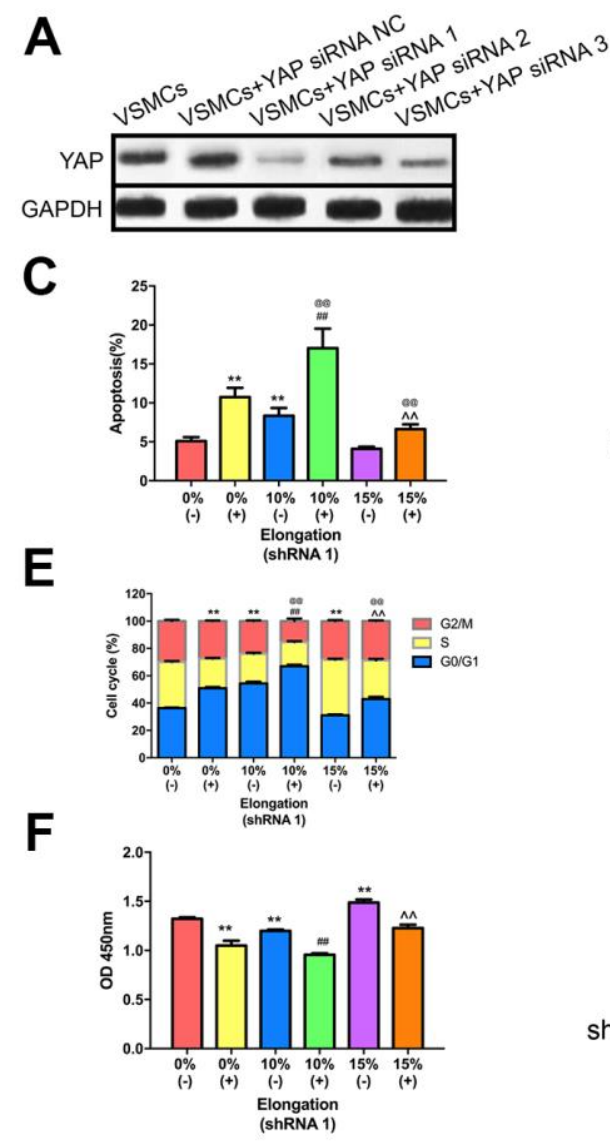

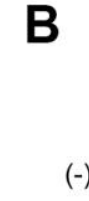

$(-)$

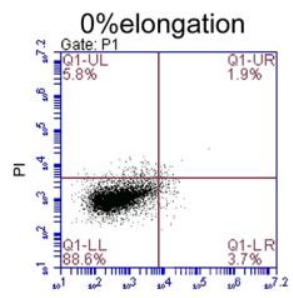
shRNA 1

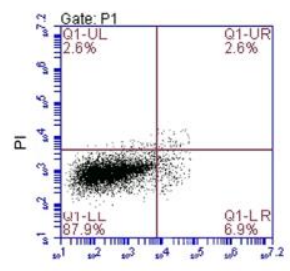

D
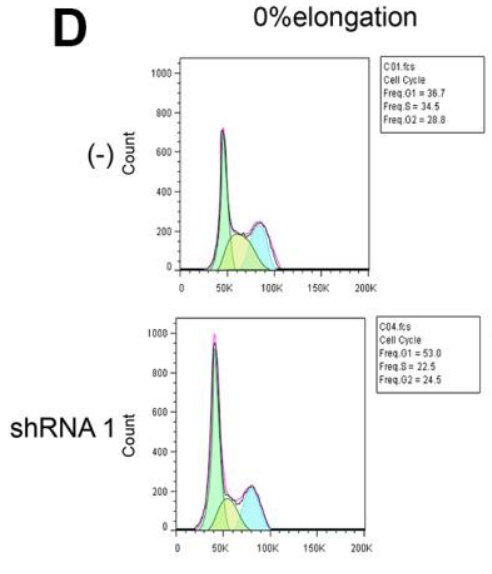
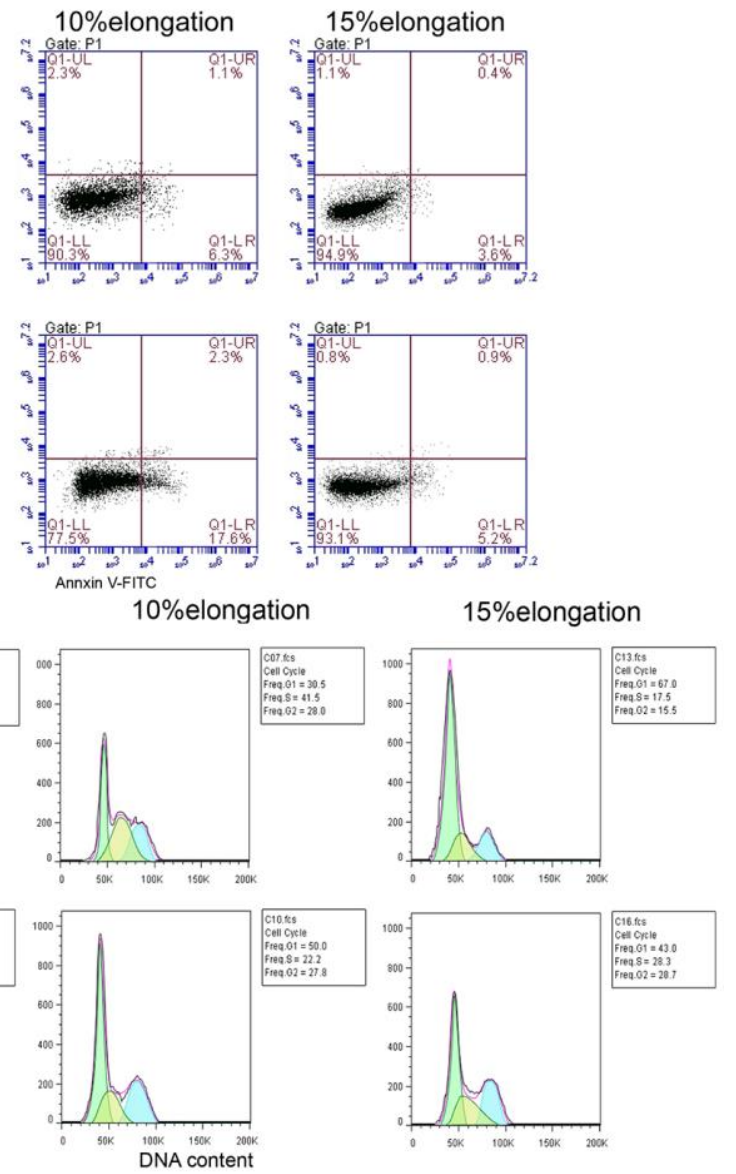

Figure 3. Knockdown of YAP impairs proliferation and apoptosis of VSMCs under different intensities of mechanical stretch. (A) The level of YAP expression in VSMCs transfected with YAP small interfering RNA (siRNA). (B) Apoptosis of VSMCs was detected by flow cytometry. (C) Quantitative analysis of apoptosis in VSMCs. (D) The cell cycle of VSMCs was detected by flow cytometry. (E) Quantitative analysis of the cell cycle in VSMCs. (F) The proliferation of VSMCs was detected by CCK-8. Values are expressed as means \pm SD. ${ }^{* *} P<0.01$, compared with $0 \%$ elongation+siRNA(-) group; ${ }^{\prime \prime} P<0.01$, compared with $10 \%$ elongation+siRNA(-) group; ${ }^{\wedge} P<0.01$, compared with $20 \%$ elongation+siRNA(-) group; @@ $P<0.01$, compared with $0 \%$ elongation+siRNA(+) group. 
stretch, indicating that more VSMCs were in a state of dormancy (Figure 3D, 3E). Compared with the 0\% control group, G0/G1 phase cells in the $10 \%$ and $15 \%$ control group were significantly increased and decreased, respectively (Figure 3D, 3E). A similar trend was apparent in the YAP shRNA groups (Figure 3D, 3E).

\section{Inhibition of the Rho-ROCK pathway affects the Hippo pathway under different intensities of stretch}

To better understand the role of the Rho-ROCK-Hippo pathway in the effects of mechanical stretch on YAP, we inhibited the Rho-ROCK pathway using Y27632. We performed immunocytochemistry staining to determine the intracellular localization of YAP. The results showed that more YAP stayed in the cytoplasm under $0 \%$ elongation following inhibition of the RhoROCK pathway (group 2) compared with 0\% elongation (group 1) (Figure 4A, 4B). Y27632 treatment combined with $10 \%$ elongation (group 4) caused a greater amount of YAP to remain in the cytoplasm compared with $10 \%$ elongation in the absence of Y27632 (group 3) (Figure 4A, 4B). The nuclear proportion of YAP following 15\% elongation with Y27623 treatment (group 6) was higher than that in groups 2-4 (Figure 4A, 4B). The nuclear proportion in group 6 was lower than that in groups 1 and 5 (Figure 4A, 4B). We then performed western blotting to assess the phosphorylation of YAP and Lats1, as well as the protein levels of ccnd1, Rho, and ROCK in the different groups. The proportion of phosphorylated YAP and phosphorylated Lats1 increased under each stretch intensity following inhibition of the Rho-ROCK pathway, while the levels of ccnd1, Rho, and ROCK decreased, suggesting that blockage of the Rho-ROCK pathway partially reduces the effect of different stretch intensities on YAP (Figure 4C).

\section{DISCUSSION}

One emerging concept is that changes in the VSMCs phenotype in response to mechanical forces are important in vascular diseases, such as hypertension, atherosclerosis, aortic aneurysms, and age-dependent arterial stiffening [21, 26, 27], in which the vascular wall is exposed to chronically elevated levels of cyclic stretch. In particular, the production of aortic aneurysms and aortic vascular VSMCs reduction are closely related. Different parts of the aorta are subject to different pressures, and the pathogenesis of aortic aneurysms may vary by location. Our study demonstrated that different intensities of stretch differently affect the proliferation and apoptosis of VSMCs. $10 \%$ and $20 \%$ of the stress-induced S phase cells reduce, may inhibit cell proliferation, $15 \%$ stressstimulated $\mathrm{S}$ phase cells relative increase, may promote cell proliferation (Figure 5). However, we believe that VSMCs will not continue to grow, or may even break up, if more than $20 \%$ elongation force is applied. This also confirmed the effect of the difference of $10 \%$ physiological elongation and $15 \%$ stress elongation on the proliferation and apoptosis of VSMCs. Therefore, we believe that applying more tension to VSMCs is not necessary. In this study we sued $10 \%$ and $15 \%$ elongation forces, which we believe are more representative experimental conditions.

Studies have found that mechanical stimulation can inhibit the Hippo pathway, increase nuclear levels of YAP, promote cell proliferation, and inhibit apoptosis. [16, 28-31] YAP is the major downstream effector of the Hippo pathway which mediates major physiological functions. YAP phosphorylation results in cytoplasmic retention, which inhibits SMC proliferation and promotes apoptosis. [32, 33] To the best of our knowledge, however, this is the first study to show that different intensities of mechanical stretch can differentially influence the proliferation and apoptosis of VSMCs through the Hippo pathway. However, our study found that different intensities of mechanical stimulation had different effects on VSMCs' proliferation and apoptosis, as well as the Hippo pathway. Stimulation with $10 \%$ elongation may activate the Hippo pathway, increasing phosphorylation of YAP and resulting in translocation of YAP into the cytoplasm, where it binds to the cytosolic protein 14-33 thereby promoting YAP degradation. These reports are consistent with our results. Our results revealed the intracellular localization of YAP under different stretch forces: $10 \%$ elongation for $6 \mathrm{~h}$ induced YAP cytoplasmic retention, while $15 \%$ elongation for $6 \mathrm{~h}$ promoted YAP entry into the nucleus. Mechanical stretching forces of $10 \%$ and $15 \%$ for $6 \mathrm{~h}$ upregulated and downregulated the phosphorylation of YAP, respectively. According to the former studies [34, 35], YAP in the nucleus, as well as the binding of YAP to the transcription factor TEAD, were decreased, leading to decreased expression of the target gene ccnd1 and inhibition of cell proliferation. When the stretching force was increased to $15 \%$, the Hippo pathway was inhibited, YAP phosphorylation was reduced, more YAP remained in the nucleus, and the transcription factor TEAD induced expression of its downstream target gene ccnd1 to promote proliferation [36-38]. Thus, $10 \%$ and $15 \%$ stimulation can affect the proliferation of arterial SMCs through the Hippo pathway. The results demonstrate that $10 \%$ mechanical stimulation promotes cell proliferation and inhibits apoptosis, while $15 \%$ mechanical stimulation had the opposite effect. This trend is consistent with previous results on the Hippo pathway [36-38]. Previous studies have shown that YAP/TAZ expression in human 


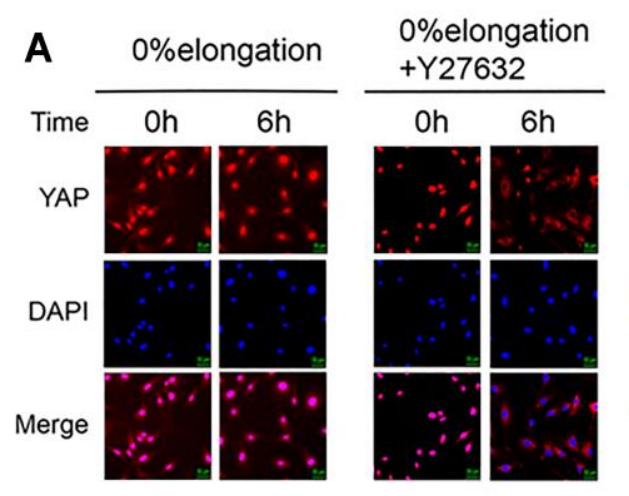

B

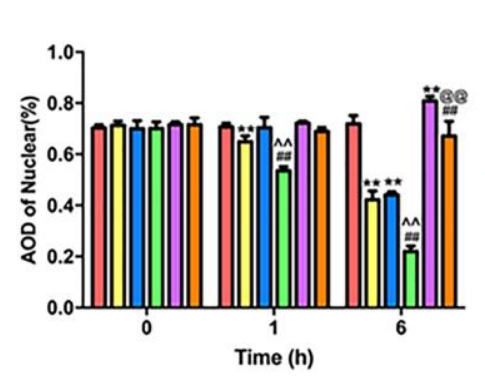

$10 \%$ elongation

\begin{tabular}{c}
\hline Oh \\
\\
\\
$\therefore$
\end{tabular}

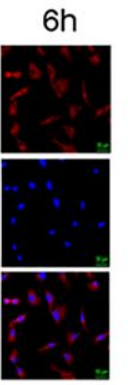

$10 \%$ elongation + Y27632

Oh $6 \mathrm{~h}$

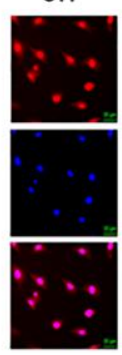

$15 \%$ elongation

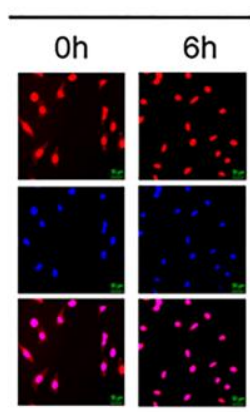

$15 \%$ elongation + Y27632

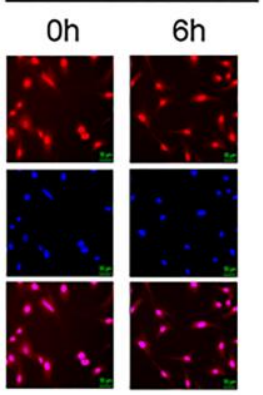

C

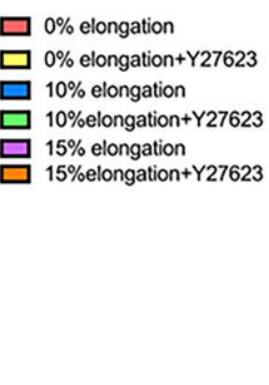

Figure 4. Inhibition of the Rho-ROCK pathway affects the Hippo pathway under different intensities of stretch. (A) The YAP localization of VSMCs was detected by immunocytochemistry. (B) Quantification of nuclear YAP amount in VSMCs. (C) The levels of phosphorylated YAP, phosphorylated Lats and ccnd1 in VSMCs were detected by western blotting. Values are expressed as means \pm SD. ${ }^{* *} P<$ 0.01 , compared with $0 \%$ elongation+siRNA(-) group; ${ }^{\# P} P<0.01$, compared with $10 \%$ elongation+siRNA(-) group; ${ }^{\wedge} P<0.01$, compared with $20 \%$ elongation+siRNA(-) group; @ $P<0.01$, compared with $0 \%$ elongation+siRNA(+) group.
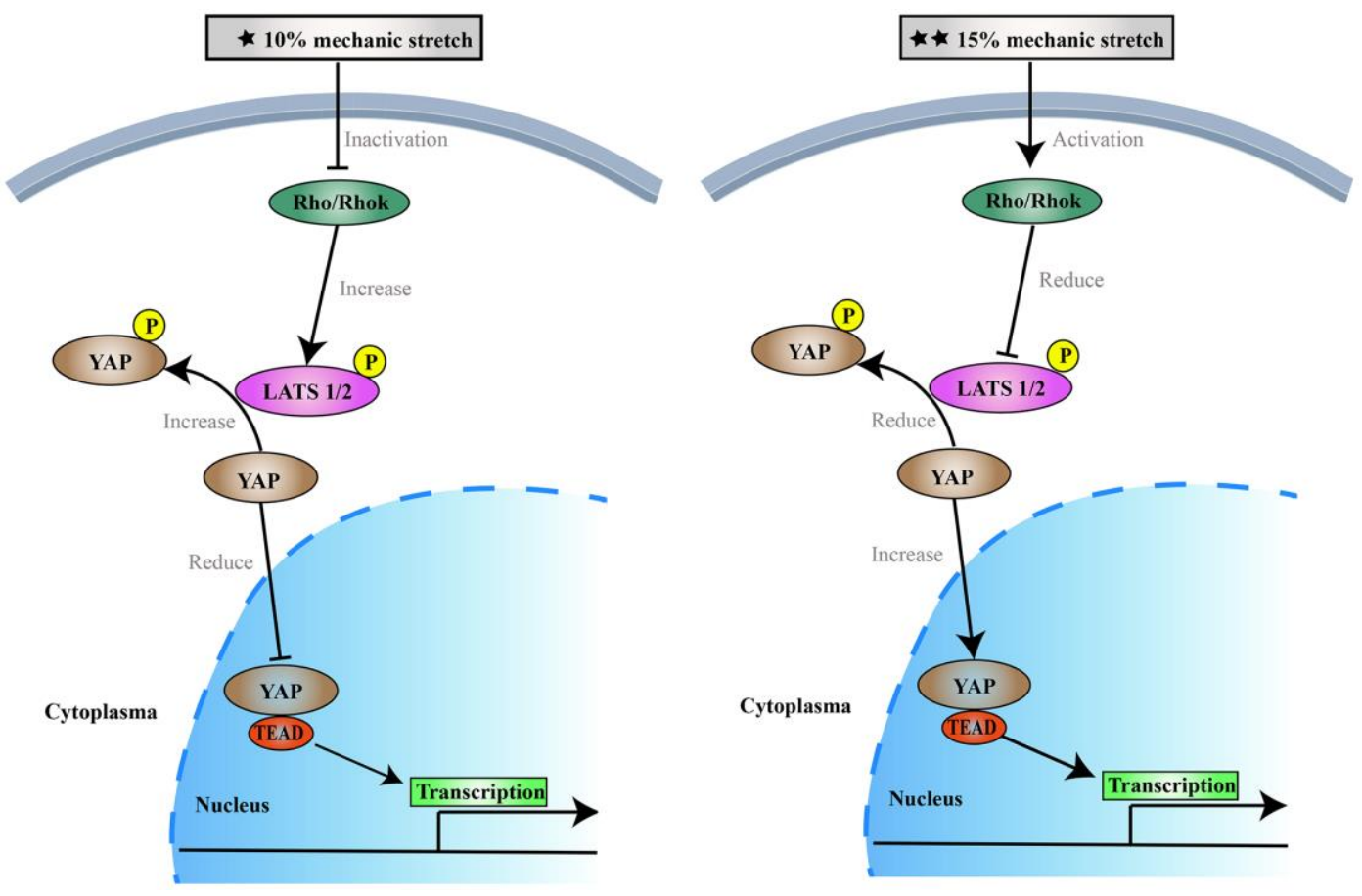

Figure 5. The effects of $10 \%$ and $15 \%$ elongation stretch on the proliferation and apoptosis of VSMCs. 
trabecular meshwork cells also varies by substrate stiffness $[39,40]$. The results of our study are consistent with previous studies investigating the effects of extracellular matrix on cells [41, 42]. After knocking down the expression of YAP, VSMCs showed the same trends in cell proliferation, apoptosis, and the cell cycle following stimulation with different intensities of stretch compared with the controls. These results indicated that different intensities of stretch stimulation could still regulate VSMCs proliferation and apoptosis following inhibition or activation of the Hippo pathway.

In addition, we tested the activity of the Rho-ROCK pathway and found that $10 \%$ elongation stimulated the expression of related proteins to decrease, while $15 \%$ elongation stimulated the expression of related proteins to increase. After blocking the Rho-ROCK pathway, the effects of different intensities of stretch on YAP were weakened and phosphorylated YAP was decreased in each group. Overall, inhibition of the Rho-ROCK pathway combined with $10 \%$ mechanical stress led to activation of the Hippo pathway, which in turn reduced nuclear localization of YAP. YAP failed to bind to TEAD; this led to a decrease in ccnd1 expression, inhibition of VSMCs proliferation, and promotion of apoptosis. When the mechanical stress was increased to $15 \%$, we observed the opposite effects.

Through our experiments, we found that the mechanism underlying the effects of mechanical stress on VSMCs is as follows: after being stimulated by physiological $10 \%$ elongation stretch, VSMCs are inhibited in various ways. Inhibition of Rho-ROCK leads to a decrease in LATS1/2 levels, which in turn leads to an increase in the phosphorylation of YAP. Phosphorylated YAP cannot enter the nucleus nor bind to TEAD. On the other hand, our ongoing experiment shows that $10 \%$ elongation stretch may downregulates the expression of miR130a, which increases the amount of VGLL4. In the nucleus, VGLL4 may competes with YAP for the opportunity to bind TEAD. As a result of these two mechanisms, $10 \%$ elongation reduced ccnd1, resulting in decreased VSMCs proliferation and increased apoptosis, while a stimulus that is lower or higher than physiological elongation resulted in opposing effects, eventually promoting the proliferation of VSMCs and reducing apoptosis.

On conclusion, our study demonstrates the possibility of preventing or treating diseases such as aortic aneurysms by controlling blood pressure. The RhoROCK pathway can be activated by mildly adjusting the blood pressure. Activation of the Rho-ROCK pathway, in turn, inhibits the Hippo pathway and ultimately promotes VSMCs proliferation and inhibits apoptosis. Our findings may provide insight into the pathogenesis prognosis of these diseases, as well as into therapeutic interventions.

\section{AUTHOR CONTRIBUTIONS}

XHW and XDL conceived and designed the experiments, LJZ, SL and ZQF analyzed and interpreted the results of the experiments, $\mathrm{CZH}, \mathrm{YGH}$ and ZSG performed the experiments.

\section{CONFLICTS OF INTEREST}

The authors declare that they have no conflicts of interest.

\section{FUNDING}

This work was supported by National International Science and Technology Cooperation Special Project [grant number 2014DFA31230; 19LBJ1005A].

\section{REFERENCES}

1. Isselbacher EM. Thoracic and abdominal aortic aneurysms. Circulation. 2005; 111:816-28. https://doi.org/10.1161/01.CIR.0000154569.08857.7A PMID:15710776

2. Tang PC, Coady MA, Lovoulos C, Dardik A, Aslan M, Elefteriades JA, Tellides G. Hyperplastic cellular remodeling of the media in ascending thoracic aortic aneurysms. Circulation. 2005; 112:1098-105. https://doi.org/10.1161/CIRCULATIONAHA.104.511717 PMID:16116068

3. Guo DC, Papke CL, Tran-Fadulu V, Regalado ES, Avidan $\mathrm{N}$, Johnson RJ, Kim DH, Pannu H, Willing MC, Sparks E, Pyeritz RE, Singh MN, Dalman RL, et al. Mutations in smooth muscle alpha-actin (ACTA2) cause coronary artery disease, stroke, and Moyamoya disease, along with thoracic aortic disease. Am J Hum Genet. 2009; 84:617-27.

https://doi.org/10.1016/j.ajhg.2009.04.007 PMID:19409525

4. Mao X, Said R, Louis H, Max JP, Bourhim M, Challande P, Wahl D, Li Z, Regnault V, Lacolley P. Cyclic stretchinduced thrombin generation by rat vascular smooth muscle cells is mediated by the integrin $\alpha v \beta 3$ pathway. Cardiovasc Res. 2012; 96:513-23. https://doi.org/10.1093/cvr/cvs274 PMID:22915765

5. Mann JM, Lam RH, Weng S, Sun Y, Fu J. A siliconebased stretchable micropost array membrane for monitoring live-cell subcellular cytoskeletal response. Lab Chip. 2012; 12:731-40. https://doi.org/10.1039/c2lc20896b PMID:22193351 
6. Hoffman BD, Grashoff C, Schwartz MA. Dynamic molecular processes mediate cellular mechanotransduction. Nature. 2011; 475:316-23. https://doi.org/10.1038/nature10316 PMID:21776077

7. Shi N, Chen SY. Mechanisms simultaneously regulate smooth muscle proliferation and differentiation. J Biomed Res. 2014; 28:40-6. https://doi.org/10.7555/JBR.28.20130130 PMID:24474962

8. Banerjee I, Carrion K, Serrano R, Dyo J, Sasik R, Lund S, Willems E, Aceves S, Meili R, Mercola M, Chen J, Zambon A, Hardiman G, et al. Cyclic stretch of embryonic cardiomyocytes increases proliferation, growth, and expression while repressing Tgf- $\beta$ signaling. J Mol Cell Cardiol. 2015; 79:133-44.

https://doi.org/10.1016/j.yjmcc.2014.11.003 PMID:25446186

9. Dai Y, Tian Y, Luo DY, Wazir R, Yue X, Li H, Wang KJ. Cyclic stretch induces human bladder smooth muscle cell proliferation in vitro through muscarinic receptors. Mol Med Rep. 2015; 11:2292-8.

https://doi.org/10.3892/mmr.2014.2984 PMID:25412212

10. Cao W, Zhang D, Li Q, Liu Y, Jing S, Cui J, Xu W, Li S, Liu J, Yu B. Biomechanical Stretch Induces Inflammation, Proliferation, and Migration by Activating NFAT5 in Arterial Smooth Muscle Cells. Inflammation. 2017; 40:2129-36.

https://doi.org/10.1007/s10753-017-0653-y

PMID:28840417

11. Felsen D, Diaz BJ, Chen J, Gonzalez J, Kristensen ML, Bohn AB, Roth BT, Poppas DP, Nørregaard R. Pressure and stretch differentially affect proliferation of renal proximal tubular cells. Physiol Rep. 2017; 5:e13346. https://doi.org/10.14814/phy2.13346 PMID:28904080

12. Mantella LE, Quan A, Verma S. Variability in vascular smooth muscle cell stretch-induced responses in 2D culture. Vasc Cell. 2015; 7:7.

https://doi.org/10.1186/s13221-015-0032-0 PMID:26301087

13. Hansen CG, Moroishi T, Guan KL. YAP and TAZ: a nexus for Hippo signaling and beyond. Trends Cell Biol. 2015; 25:499-513.

https://doi.org/10.1016/i.tcb.2015.05.002 PMID:26045258

14. Wada K, Itoga K, Okano T, Yonemura S, Sasaki H. Hippo pathway regulation by cell morphology and stress fibers. Development. 2011; 138:3907-14. https://doi.org/10.1242/dev.070987 PMID:21831922

15. Halder G, Dupont S, Piccolo S. Transduction of mechanical and cytoskeletal cues by YAP and TAZ. Nat Rev Mol Cell Biol. 2012; 13:591-600. https://doi.org/10.1038/nrm3416 PMID:22895435

16. Codelia VA, Sun G, Irvine KD. Regulation of YAP by mechanical strain through Jnk and Hippo signaling. Curr Biol. 2014; 24:2012-7. https://doi.org/10.1016/i.cub.2014.07.034 PMID:25127217

17. Mohri Z, Del Rio Hernandez A, Krams R. The emerging role of YAP/TAZ in mechanotransduction. J Thorac Dis. 2017; 9:E507-9.

https://doi.org/10.21037/jtd.2017.03.179 PMID:28616323

18. Harvey K, Tapon N. The Salvador-Warts-Hippo pathway - an emerging tumour-suppressor network. Nat Rev Cancer. 2007; 7:182-91. https://doi.org/10.1038/nrc2070 PMID:17318211

19. Pan D. The hippo signaling pathway in development and cancer. Dev Cell. 2010; 19:491-505. https://doi.org/10.1016/i.devcel.2010.09.011 PMID:20951342

20. Zhao B, Li L, Lei Q, Guan KL. The Hippo-YAP pathway in organ size control and tumorigenesis: an updated version. Genes Dev. 2010; 24:862-74. https://doi.org/10.1101/gad.1909210 PMID:20439427

21. Dupont S, Morsut L, Aragona M, Enzo E, Giulitti S, Cordenonsi M, Zanconato F, Le Digabel J, Forcato $M$, Bicciato S, Elvassore N, Piccolo S. Role of YAP/TAZ in mechanotransduction. Nature. 2011; 474:179-83. https://doi.org/10.1038/nature10137 PMID:21654799

22. Mao B, Gao Y, Bai Y, Yuan Z. Hippo signaling in stress response and homeostasis maintenance. Acta Biochim Biophys Sin (Shanghai). 2015; 47:2-9.

https://doi.org/10.1093/abbs/gmu109 PMID:25476206

23. Shi X, Yin Z, Ling B, Wang L, Liu C, Ruan X, Zhang W, Chen L. Rho differentially regulates the Hippo pathway by modulating the interaction between Amot and Nf2 in the blastocyst. Development. 2017; 144:3957-67.

https://doi.org/10.1242/dev.157917 PMID:28947533

24. Kono K, Tamashiro DA, Alarcon VB. Inhibition of RHOROCK signaling enhances ICM and suppresses TE characteristics through activation of Hippo signaling in the mouse blastocyst. Dev Biol. 2014; 394:142-55. https://doi.org/10.1016/j.ydbio.2014.06.023 PMID:24997360

25. Huang J, Li LS, Yang DL, Gong QH, Deng J, Huang XN. Inhibitory Effect of Ginsenoside Rg1 on Vascular Smooth Muscle Cell Proliferation Induced by PDGF-BB Is Involved in Nitric Oxide Formation. Evid Based Complement Alternat Med. 2012; 2012:314395. https://doi.org/10.1155/2012/314395 PMID:22474498 
26. Qiu H, Zhu Y, Sun Z, Trzeciakowski JP, Gansner M, Depre C, Resuello RR, Natividad FF, Hunter WC, Genin GM, Elson EL, Vatner DE, Meininger GA, Vatner SF. Short communication: vascular smooth muscle cell stiffness as a mechanism for increased aortic stiffness with aging. Circ Res. 2010; 107:615-9.

https://doi.org/10.1161/CIRCRESAHA.110.221846

PMID:20634486

27. Lacolley P, Regnault V, Nicoletti A, Li Z, Michel JB. The vascular smooth muscle cell in arterial pathology: a cell that can take on multiple roles. Cardiovasc Res. 2012; 95:194-204.

https://doi.org/10.1093/cvr/cvs135 PMID:22467316

28. Cui Y, Hameed FM, Yang B, Lee K, Pan CQ, Park S, Sheetz M. Cyclic stretching of soft substrates induces spreading and growth. Nat Commun. 2015; 6:6333. https://doi.org/10.1038/ncomms7333 PMID:25704457

29. Shafieyan $Y$, Hinz B. Signs of stress on soft surfaces : A commentary on: Cui, Y., F.M. Hameed, B. Yang, K. Lee, C.Q. Pan, S. Park, and M. Sheetz. 2015. Cyclic stretching of soft substrates induces spreading and growth. Nat Commun. 6:6333. J Cell Commun Signal. 2015; 9:305-7.

https://doi.org/10.1007/s12079-015-0305-7 PMID:26277348

30. Endlich K, Kliewe F, Endlich N. Stressed podocytesmechanical forces, sensors, signaling and response. Pflugers Arch. 2017; 469:937-49.

https://doi.org/10.1007/s00424-017-2025-8

PMID:28687864

31. Liu T, Xu J, Guo JL, Lin CY, Luo WM, Yuan Y, Liu H, Zhang J. YAP1 up-regulation inhibits apoptosis of aortic dissection vascular smooth muscle cells. Eur Rev Med Pharmacol Sci. 2017; 21:4632-9.

PMID:29131252

32. Harvey KF, Pfleger CM, Hariharan IK. The Drosophila Mst ortholog, hippo, restricts growth and cell proliferation and promotes apoptosis. Cell. 2003; 114:457-67.

https://doi.org/10.1016/s0092-8674(03)00557-9 PMID:12941274

33. Liu W, Wu J, Xiao L, Bai Y, Qu A, Zheng Z, Yuan Z. Regulation of neuronal cell death by c-Abl-Hippo/MST2 signaling pathway. PLoS One. 2012; 7:e36562. https://doi.org/10.1371/journal.pone.0036562 PMID:22590567

34. Xie Q, Chen J, Feng H, Peng S, Adams U, Bai Y, Huang L, Li J, Huang J, Meng S, Yuan Z. YAP/TEAD-mediated transcription controls cellular senescence. Cancer Res. 2013; 73:3615-24. https://doi.org/10.1158/0008-5472.CAN-12-3793 PMID:23576552
35. Wei H, Wang F, Wang Y, Li T, Xiu P, Zhong J, Sun X, Li J. Verteporfin suppresses cell survival, angiogenesis and vasculogenic mimicry of pancreatic ductal adenocarcinoma via disrupting the YAP-TEAD complex. Cancer Sci. 2017; 108:478-87.

https://doi.org/10.1111/cas.13138 PMID:28002618

36. Yao $M$, Wang $Y$, Zhang $P$, Chen $H, X u ~ Z$, Jiao J, Yuan Z. $B M P 2-S M A D$ signaling represses the proliferation of embryonic neural stem cells through YAP. J Neurosci. 2014; 34:12039-48.

https://doi.org/10.1523/JNEUROSCI.0486-14.2014 PMID:25186749

37. Mizuno T, Murakami H, Fujii M, Ishiguro F, Tanaka I, Kondo Y, Akatsuka S, Toyokuni S, Yokoi K, Osada H, Sekido Y. YAP induces malignant mesothelioma cell proliferation by upregulating transcription of cell cyclepromoting genes. Oncogene. 2012; 31:5117-22.

https://doi.org/10.1038/onc.2012.5 PMID:22286761

38. Benhaddou A, Keime C, Ye T, Morlon A, Michel I, Jost B, Mengus G, Davidson I. Transcription factor TEAD4 regulates expression of myogenin and the unfolded protein response genes during $\mathrm{C} 2 \mathrm{C} 12$ cell differentiation. Cell Death Differ. 2012; 19:220-31.

https://doi.org/10.1038/cdd.2011.87 PMID:21701496

39. Raghunathan VK, Morgan JT, Dreier B, Reilly CM, Thomasy SM, Wood JA, Ly I, Tuyen BC, Hughbanks M, Murphy $\mathrm{CJ}$, Russell P. Role of substratum stiffness in modulating genes associated with extracellular matrix and mechanotransducers YAP and TAZ. Invest Ophthalmol Vis Sci. 2013; 54:378-86.

https://doi.org/10.1167/iovs.12-11007 PMID:23258147

40. Thomasy SM, Morgan JT, Wood JA, Murphy CJ, Russell $P$. Substratum stiffness and latrunculin B modulate the gene expression of the mechanotransducers YAP and TAZ in human trabecular meshwork cells. Exp Eye Res. 2013; 113:66-73.

https://doi.org/10.1016/i.exer.2013.05.014 PMID:23727052

41. Levental KR, Yu H, Kass L, Lakins JN, Egeblad M, Erler JT, Fong SF, Csiszar K, Giaccia A, Weninger W, Yamauchi M, Gasser DL, Weaver VM. Matrix crosslinking forces tumor progression by enhancing integrin signaling. Cell. 2009; 139:891-906.

https://doi.org/10.1016/i.cell.2009.10.027

PMID:19931152

42. Engler AJ, Sen S, Sweeney HL, Discher DE. Matrix elasticity directs stem cell lineage specification. Cell. 2006; 126:677-89. https://doi.org/10.1016/i.cell.2006.06.044 PMID:16923388 